\title{
Risk for Deep Fungal Infections During IL-17 and IL-23 Inhibitor Therapy for Psoriasis
}

\author{
Michael P. Lee, MD; Kevin K. Wu, MD; Erica B. Lee, MD; Jashin J. Wu, MD
}

\section{PRACTICE POINTS}

- The use of IL-17, IL-12/IL-23, and IL-23 inhibitors for psoriasis and other inflammatory conditions does not appear to increase the risk for deep fungal infections.

- Physicians should still be cautiously optimistic in prescribing these medications, as IL-17 and IL-23 play a central role in immunologic defenses, particularly against fungi.

- A high index of suspicion should be maintained for patients from endemic areas who are being treated with biologics.
Psoriasis is an inflammatory disease with both skin and joint manifestations. Focused biologics have been developed to target specific cytokines implicated in psoriasis and are becoming increasingly utilized. Recently, the advent of newer biologics, including IL-17, IL-12/IL-23, and IL-23 inhibitors, have garnered interest as promising treatments for psoriasis and other inflammatory conditions. Although IL-17 and IL-23 have been studied in the pathophysiology of psoriasis, they also play a central role in immunologic defenses, including those against fungi. Therefore, use of these interleukin inhibitors may theoretically impair the immune system against deep fungal infections. We reviewed the available literature investigating the risk for invasive fungal infections in patients treated with IL-17 and IL-23 inhibitors for psoriasis or other inflammatory conditions. Randomized controlled trials (RCTs), including extended trials and clinical trials, were reviewed, and we found that although there was a small number of patients who developed superficial candidiasis, there were no reports of invasive fungal disease. Although these results support the safety and the low risk for deep fungal infection with these biologics, caution is still warranted, as these medications are relatively new. Appropriate screening and management of fungal disease should still be practiced when utilizing these medications in the treatment of psoriasis and other inflammatory conditions.

Cutis. 2020;106:199-205.

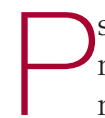
soriasis is a common chronic, multisystem, inflammatory disease with predominantly skin and joint manifestations that affects approximately $2 \%$ of the world's population. ${ }^{1}$ It occurs in a variety of clinical forms, from a few well-demarcated, erythematous plaques with a silvery scale to involvement of almost the entire body surface area. Beyond the debilitating physical ailments of the disease, psoriasis also may have psychosocial effects on quality of life. ${ }^{2}$ The pathogenesis of psoriasis is not fully understood but represents a complex multifactorial disease with both immune-mediated and genetic components. Characterized by hyperplasia of epidermal keratinocytes, psoriasis is shown to be mediated by infiltration of T-cell lymphocytes with an increase of various inflammatory cytokines, including tumor necrosis factor (TNF) $\alpha .{ }^{3}$ More recently, interactions of helper T cells $\left(\mathrm{T}_{\mathrm{H}} 17\right)$ via IL-17 and IL-23 have been supported to play a major role in the pathogenesis of psoriasis. ${ }^{4,5}$

With the growing understanding of the pathophysiology of psoriasis, focused biologics have been developed

Drs. M.P. Lee and K.K. Wu are from the Department of Internal Medicine, University of California, Irvine, Orange. Dr. E.B. Lee is from the Department of Internal Medicine, Santa Barbara Cottage Hospital, California. Dr. J.J. Wu is from the Dermatology Research and Education Foundation, Irvine, California. Drs. M.P. Lee, K.K. Wu, and E.B. Lee report no conflict of interest. Dr. J.J. Wu is or has been an consultant, investigator, or speaker for AbbVie Inc; Almirall; Amgen; Arcutis Biotherapeutics; Boehringer Ingelheim; Bristol Myers Squibb; Dermavant Sciences Ltd; Dr. Reddy's Laboratories; Eli Lilly and Company; Galderma; Janssen Pharmaceuticals, Inc; LEO Pharma; Novartis; Regeneron Pharmaceuticals; Sanofi Genzyme; Sun Pharmaceutical Industries Ltd; UCB; and Valeant Pharmaceuticals North America LLC.

The eTable is available in the Appendix online at www.mdedge.com/dermatology.

Correspondence: Jashin J. Wu, MD (jashinwu@gmail.com).

doi:10.12788/cutis.0088 
to target specific cytokines implicated in the disease process and have been increasingly utilized. Tumor necrosis factor $\alpha$ inhibitors, including adalimumab, infliximab, and etanercept, along with the IL-12/IL-23 inhibitor ustekinumab, have been revolutionary in psoriasis treatment by providing safe and effective long-term therapy; however, there is concern of life-threatening infections with biologics because of the immunosuppressive effects and mechanisms of action. ${ }^{6}$ Specifically, there have been reported cases of deep fungal infections associated with TNF- $\alpha$ inhibitor use. ${ }^{7}$

Recently, the advent of IL-17 and IL-23 inhibitors has garnered notable interest in these biologics as promising treatments for psoriasis. With IL-17 and IL-23 supported to have a major role in the pathogenesis of psoriasis, targeting the cytokine is not only logical but also has proven to be effacacious. ${ }^{8-10}$ Secukinumab, ixekizumab, and brodalumab are IL-17 inhibitors that have been approved by the US Food and Drug Administration (FDA) for the treatment of psoriasis. Secukinumab and ixekizumab are anti-IL-17A monoclonal antibodies, whereas brodalumab is an anti-IL-17 receptor antibody. Risankizumab, guselkumab, and tildrakizumab are IL-23 inhibitors that also have been approved by the FDA for the treatment of psoriasis. As with older biologics, there is concern over the safety of these inhibitors because of the central role of IL-17 and IL-23 in both innate and adaptive immune responses, particularly against fungi. ${ }^{11}$ Therefore, use of biologics targeting IL-17 and IL-23 may increase susceptibility to deep fungal infections.

Safety data and discussion of the risk for deep fungal infections from IL-17, IL-12/IL-23, and IL-23 inhibitor use for psoriasis treatment currently are lacking. Given the knowledge gap, we sought to synthesize and review the current evidence on risks for deep fungal infections during biologic therapy in patients with psoriasis, with a focus on IL-17 inhibitor therapies.

\section{METHODS}

A PubMed search of articles indexed for MEDLINE from database inception to 2019 (1946-2019) was performed to find randomized controlled trials (RCTs), including extended trials and clinical trials, for IL-17, IL-12/IL-23, and IL-23 inhibitors approved by the FDA for psoriasis treatment. The following keywords were used: psoriasis or inflammatory disease and secukinumab, ixekizumab, brodalumab, ustekinumab, risankizumab, guselkumab, or tildrakizumab. Studies were restricted to the English-language literature, and those that did not provide adequate safety data on the specific types of infections that occurred were excluded.

\section{RESULTS \\ IL-17 Inhibitors}

Our search yielded RCTs, some including extension trials, and clinical trials of IL-17 inhibitors used for psoriatic disease and other nonpsoriatic conditions (Table).
Risk for Deep Fungal Infection With Secukinumab-The queried studies included 20 RCTs or clinical trials along with extension trials of 3746 patients with psoriasis or other inflammatory conditions, with follow-up ranging from 12 to 52 weeks. In a 3-year extension study of SCULPTURE, Bissonnette et $\mathrm{al}^{12}$ reported no new safety concerns for the 340 patients with moderate to severe psoriasis treated with secukinumab. Common adverse events (AEs) included nasopharyngitis, upper respiratory tract infections, and headache, but there were no reports of deep fungal infections. ${ }^{12}$ In a subsequent 5 -year analysis of 168 patients that focused on the 300-mg fixed interval treatment with secukinumab, the safety profile remained favorable, with 0 reports of invasive fungal infections. ${ }^{13}$ A study (FEATURE) of 118 patients with psoriasis treated with a prefilled syringe of 300 or $150 \mathrm{mg}$ of secukinumab also described an acceptable safety profile and reported no deep fungal infections. ${ }^{14}$ JUNCTURE, another study utilizing autoinjectors, also found that treatment with 300 or $150 \mathrm{mg}$ of secukinumab was well tolerated in 121 patients, with no deep fungal infections. ${ }^{15}$ Common AEs for both studies included nasopharyngitis and headache. ${ }^{14,15}$ A 24-week phase 3 study for scalp psoriasis treated with secukinumab also reported 0 deep fungal infections in 51 patients. ${ }^{16}$ In an RCT comparing secukinumab and ustekinumab for moderate to severe plaque psoriasis, Blauvelt et $\mathrm{a}^{17}$ demonstrated that the incidence of serious AEs was comparable between the 2 groups, with no reports of invasive fungal infections in the 334 patients exposed to secukinumab. The CLEAR study, which compared secukinumab and ustekinumab, also found no reported deep fungal disease in the 335 patients exposed to secukinumab. ${ }^{18}$ Secukinumab exhibited a similar safety profile to ustekinumab in both studies, with common AEs being headache and nasopharyngitis. ${ }^{17,18}$ The GESTURE study investigated the efficacy of secukinumab in 137 patients with palmoplantar psoriasis and reported a favorable profile with no reports of deep fungal disease. ${ }^{19}$ In a subanalysis of the phase 3 study ERASURE, secukinumab was shown to have a robust and sustainable efficacy in 58 Japanese patients with moderate to severe plaque psoriasis, and there were no reports of invasive fungal infections. ${ }^{20}$ Another subanalysis of 36 Taiwanese patients from the ERASURE study also had similar findings, with no dose relationship observed for AEs. ${ }^{21}$ In a phase 2 study of 103 patients with psoriasis, Papp et $\mathrm{al}^{22}$ demonstrated $\mathrm{AE}$ rates that were similar across different doses of secukinumab-3×150 mg, $3 \times 75 \mathrm{mg}$, $3 \times 25 \mathrm{mg}$, and $1 \times 25 \mathrm{mg}$ - and described no incidences of invasive fungal disease. In a phase 2 regimen-finding study of 337 patients conducted by Rich et $\mathrm{al}^{23}$ the most commonly reported AEs included nasopharyngitis, worsening psoriasis, and upper respiratory tract infections, but there were no reported deep fungal infections.

Our search also resulted in studies specific to the treatment of psoriatic arthritis (PsA) with secukinumab. 


\section{IL-17 Inhibitor Exposure Data Summary}

\begin{tabular}{|c|c|c|c|c|c|c|}
\hline Reference & Year & Country & IL-17 Inhibitor & $\begin{array}{l}\text { Patients } \\
\text { Exposed to IL-17 } \\
\text { Inhibitor, n }\end{array}$ & Data Source & $\begin{array}{l}\text { Reported } \\
\text { Deep Fungal } \\
\text { Infections, n }\end{array}$ \\
\hline Bissonnette et $\mathrm{al}^{12}$ & 2017 & International & Secukinumab & 340 & Extension & 0 \\
\hline Bissonnette et $\mathrm{al}^{13}$ & 2018 & International & Secukinumab & 168 & Extension & 0 \\
\hline Blauvelt et $\mathrm{al}^{14}$ & 2015 & International & Secukinumab & 118 & RCT & 0 \\
\hline Paul et al ${ }^{15}$ & 2015 & International & Secukinumab & 121 & $\mathrm{RCT}$ & 0 \\
\hline Bagel et al16 & 2017 & United States & Secukinumab & 51 & $\mathrm{RCT}$ & 0 \\
\hline Blauvelt et al ${ }^{17}$ & 2017 & International & Secukinumab & 334 & Clinical trial & 0 \\
\hline Thaci et $a^{18}$ & 2015 & International & Secukinumab & 335 & Clinical trial & 0 \\
\hline Gottlieb et $\mathrm{al}^{19}$ & 2017 & International & Secukinumab & 137 & $\mathrm{RCT}$ & 0 \\
\hline Ohtsuki et al20 & 2014 & Japan & Secukinumab & 58 & RCT & 0 \\
\hline Wu et $\mathrm{al}^{21}$ & 2017 & Taiwan & Secukinumab & 36 & I & 0 \\
\hline Papp et a $\left.\right|^{22}$ & 2013 & International & Secukinumab & 103 & $\mathrm{RCT}$ & 0 \\
\hline Rich et $\mathrm{al}^{23}$ & 2013 & International & Secukinumab & 337 & $\mathrm{RCT}$ & 0 \\
\hline Mclnnes et al ${ }^{9}$ & 2014 & International & Secukinumab & 28 & $\mathrm{RCT}$ & 0 \\
\hline Kavanaugh et $\mathrm{a}^{24}$ & 2017 & International & Secukinumab & 404 & RCT & 0 \\
\hline Mclnnes et al ${ }^{25}$ & 2015 & International & Secukinumab & 299 & RCT & 0 \\
\hline Nash et al26 & 2018 & International & Secukinumab & 277 & RCT & 0 \\
\hline Sticherling et $\mathrm{al}^{27}$ & 2017 & International & Secukinumab & 105 & Clinical trial & 0 \\
\hline Braun et $\mathrm{a}^{28}$ & 2017 & International & Secukinumab & 200 & $\mathrm{RCT}$ & 0 \\
\hline Marzo-Ortega et al ${ }^{29}$ & 2017 & International & Secukinumab & 145 & $\mathrm{RCT}$ & 0 \\
\hline Pavelka et al ${ }^{30}$ & 2017 & International & Secukinumab & 150 & $\mathrm{RCT}$ & 0 \\
\hline Callis Duffin et $\mathrm{al}^{31}$ & 2017 & United States & Ixekizumab & 204 & $\mathrm{RCT}$ & 0 \\
\hline Gordon et $\mathrm{al}^{32}$ & 2016 & International & Ixekizumab & 2334 & $\mathrm{RCT}$ & 0 \\
\hline Saeki et al33 & 2017 & Japan & Ixekizumab & 91 & Clinical trial & 0 \\
\hline Reich et al ${ }^{34}$ & 2017 & International & Ixekizumab & 136 & Clinical Trial & 0 \\
\hline Zachariae et al ${ }^{35}$ & 2018 & International & Ixekizumab & 211 & Clinical trial & 0 \\
\hline van der Heijde et al ${ }^{36}$ & 2018 & International & Ixekizumab & 381 & $\mathrm{RCT}$ & 0 \\
\hline van der Heijde et al ${ }^{37}$ & 2018 & International & Ixekizumab & 164 & $\mathrm{RCT}$ & 0 \\
\hline Nakagawa et al ${ }^{38}$ & 2016 & Japan & Brodalumab & 113 & $\mathrm{RCT}$ & 0 \\
\hline Umezawa et al ${ }^{39}$ & 2016 & Japan & Brodalumab & 145 & Clinical trial & 0 \\
\hline Papp et $\mathrm{al}^{10}$ & 2012 & International & Brodalumab & 320 & $\mathrm{RCT}$ & 0 \\
\hline Papp et $a^{140}$ & 2016 & International & Brodalumab & 441 & $\mathrm{RCT}$ & 0 \\
\hline Papp et al ${ }^{41}$ & 2014 & International & Brodalumab & 181 & Clinical trial & 0 \\
\hline Yamasaki et al42 & 2017 & Japan & Brodalumab & 30 & Clinical trial & 0 \\
\hline Mease et $\mathrm{al}^{43}$ & 2014 & International & Brodalumab & 113 & RCT & 0 \\
\hline Martin et al ${ }^{44}$ & 2013 & International & Brodalumab & 30 & $\mathrm{RCT}$ & 0 \\
\hline Busse et al ${ }^{45}$ & 2013 & International & Brodalumab & 226 & RCT & 0 \\
\hline
\end{tabular}

Abbreviation: RCT, randomized controlled trial. 
McInnes et $\mathrm{al}^{9}$ conducted a phase 2 proof-of-concept trial for patients with PsA and reported no deep fungal infections in 28 patients exposed to $10 \mathrm{mg} / \mathrm{kg}$ of secukinumab. A 2-year follow-up with the cohort from FUTURE 1, a phase 3 clinical trial, also showed no new or unexpected safety signals in 404 patients exposed to 150 or $75 \mathrm{mg}$ of secukinumab, including no reports of invasive fungal disease. ${ }^{24}$ FUTURE 2, a phase 3 clinical trial, demonstrated that the most common $\mathrm{AE}$ was upper respiratory tract infection in the 299 patients treated with secukinumab, but there were no recorded invasive fungal infections. ${ }^{25}$ In FUTURE 3, 277 patients were treated with secukinumab, with 14 nonserious candida infections but no observed deep fungal infections. ${ }^{26} \mathrm{~A}$ study comparing secukinumab to fumaric acid esters reported that 6 of 105 patients treated with secukinumab also experienced superficial candidiasis, but there were no reports of deep fungal disease. ${ }^{27}$

Secukinumab also has been used in the treatment of ankylosing spondylitis in a phase 3 RCT (MEASURE 1) in which 4 cases of superficial candidiasis were reported (0.7 cases per 100 patient-years of secukinumab) that were all resolved with standard antifungal therapy. ${ }^{28} \mathrm{In}$ MEASURE 2, a 5-year phase $3 \mathrm{RCT}, 145$ patients were treated with secukinumab for ankylosing spondylitis, with common AEs including nasopharyngitis, diarrhea, and upper respiratory tract infection, but there were no reports of any invasive fungal infections. ${ }^{29}$ MEASURE 3 also demonstrated similar results in which no invasive fungal infections were observed. ${ }^{30}$

Risk for Deep Fungal Infection With IxekizumabThe queried studies included 7 RCTs or clinical trials of 3523 patients with psoriasis or other inflammatory conditions, with follow-up ranging from 12 to 52 weeks. In UNCOVER-A, a phase 3 RCT of the pharmacokinetics and safety of ixekizumab, 204 patients were randomized to a prefilled syringe or autoinjector; $48 \%$ of patients experienced AEs, but no invasive fungal infections were observed. ${ }^{31}$ In an analysis of 3 phase 3 trials of ixekizumab including a total 2334 patients treated with ixekizumab from UNCOVER-1, UNCOVER-2, and UNCOVER-3, oral candidiasis frequently was reported, but no candidal infections met criteria for serious invasive infection. ${ }^{32}$ In UNCOVER-J, a 52-week phase 3 open-label trial of Japanese patients, 91 patients were treated for plaque psoriasis, erythrodermic psoriasis, or generalized pustular psoriasis using ixekizumab; the most common AEs included allergic reactions and injection-site reactions. One case of oral candidiasis was reported, but there were no reported cases of invasive fungal infections. ${ }^{33}$ A comparison of ixekizumab vs ustekinumab from the IXORA-S trial demonstrated no substantial differences in AEs between the two, and no cases of deep fungal infections were reported. The most common AE between the 2 groups was nasopharyngitis. ${ }^{34}$ An open-label extension over 4 years of a phase 2 RCT treated 211 patients with either 120 or $80 \mathrm{mg}$ of ixekizumab; $87 \%$ of patients had experienced at least $1 \mathrm{AE}$, and all AEs were considered mild or moderate in severity, with no invasive fungal disease. ${ }^{35}$

Our search also resulted in 1 study specific to the treatment of PsA with ixekizumab. A phase 3, 52-week study of patients treated with ixekizumab for PsA observed 2 incidences of oral candidiasis and nail candida infections, but no invasive fungal infections were reported. ${ }^{36}$

We also found 1 study of ixekizumab used in the treatment of ankylosing spondylitis. COAST-V was a phase 3 RCT of patients treated for ankylosing spondylitis in which 164 patients were treated with ixekizumab; no serious AEs were recorded, including 0 deep fungal infections. The most common AEs observed were nasopharyngitis and upper respiratory tract infections. ${ }^{37}$

Risk for Deep Fungal Infection With Brodalumab-The queried studies included 9 RCTs and 3 clinical trials along with extension trials of 1599 patients with psoriasis or other inflammatory conditions, with follow-up ranging from 12 to 120 weeks. In a phase 2 RCT of Japanese patients with moderate to severe plaque psoriasis, 113 patients were treated with 70,140, or $210 \mathrm{mg}$ of brodalumab, and the most common AEs were nasopharyngitis, diarrhea, and upper respiratory tract inflammation. There were no reported cases of fungal infections in the study. ${ }^{38}$ In an open-label extension study of Japanese patients that evaluated the long-term clinical safety of brodalumab, 145 patients were enrolled and observed similar AEs to the RCT, with 7 patients experiencing oral candidiasis and 1 patient having skin candidiasis, but there were no observed deep fungal infections. ${ }^{39}$ In AMG 827, which evaluated the efficacy and safety of brodalumab, 320 patients were treated, and only 2 serious AEs were reported, neither of which were deep fungal disease. ${ }^{10} \mathrm{~A}$ phase $3 \mathrm{RCT}$ conducted by Papp et al ${ }^{40}$ (AMAGINE-1) also treated 441 patients with moderate to severe plaque psoriasis with brodalumab and observed candida infections in 9 patients that were mild to moderate and responsive to treatment, with no patients discontinuing the study. In a 120-week open-label extension study of 181 patients, Papp et $\mathrm{a}^{41}$ reported $8 \%$ of patients experienced serious AEs, with 1 case of latent tuberculosis that led to withdrawal of treatment. A study also investigated the efficacy and safety of brodalumab in 30 patients with generalized pustular psoriasis or psoriatic erythroderma and observed 2 cases of mild candida infections that resolved with treatment. There were no reports of invasive fungal disease. ${ }^{42}$

Our search also resulted in studies of brodalumab used in the treatment of PsA and nonpsoriatic diseases. In one phase $2 \mathrm{RCT}, 113$ patients with PsA were treated with $140 \mathrm{mg}$, $280 \mathrm{mg}$, or combined doses of brodalumab, with the most common AEs being nasopharyngitis, upper respiratory tract infection, and diarrhea, but there were no reports of deep fungal infection. ${ }^{43}$ In a phase $1 \mathrm{~b}$ trial of patients with methotrexate-resistant rheumatoid arthritis treated with brodalumab, common AEs 
reported included headache, cough, and abdominal pain, with only 1 case of oral candidiasis that was determined not to be drug related. ${ }^{44}$ Finally, an RCT of patients with moderate to severe asthma treated 226 patients with brodalumab and reported a greater incidence of oral candidiasis in treatment groups compared with placebo $(3.5 \%$ vs $0 \%)$ but saw no instances of invasive fungal infection. ${ }^{45}$

\section{IL-12/IL-23 Inhibitor}

Risk for Deep Fungal Infection With Ustekinumab-The queried studies included 4 RCTs of 954 patients with psoriasis treated with ustekinumab (eTable). ${ }^{46-49}$ Within these trials, there were no reported cases of serious infections involving deep fungal organisms during the stated follow-up period. The literature search also found long-term safety data from the ACCEPT and PHOENIX trials that included 5437 patients with psoriasis treated with ustekinumab. ${ }^{66,67}$ There also were no demonstrated incidences of invasive fungal disease in these studies, with most cases of infection being common bacterial or viral infections.

\section{IL-23 Inhibitors}

Risk for Deep Fungal Infection With Risankizumab, Guselkumab, and Tildrakizumab -The queried studies included 16 RCTs or clinical trials for psoriatic patients treated with IL-23 inhibitors, including 5 with risankizumab, ${ }^{50-54} 9$ with guselkumab $^{55-63}$ and 2 with tildrakizumab. ${ }^{64,65}$ Within these trials there were no observed cases of serious infections with deep fungal disease.

\section{COMMENT}

Our literature review has demonstrated that there does not appear to be an increased incidence of deep fungal infections for patients treated with IL-17, IL-12/IL-23, or IL-23 inhibitors for psoriatic disease. All of the reviewed studies found no cases of invasive fungal infections for patients with psoriasis treated with secukinumab, ixekizumab, brodalumab, ustekinumab, risankizumab, guselkumab, or tildrakizumab. Patients with other inflammatory conditions, such as ankylosing spondylitis, rheumatoid arthritis, and asthma, also did not appear to show an increased incidence of deep fungal disease.

Although these results show promising safety data for the use of these biologic therapies in treating inflammatory conditions, caution still is warranted, as these medications still are relatively new, with FDA approvals within the last 5 years. Safety data among different study populations also cannot be derived without further investigation, and much of the available literature is limited in long-term data. More extended trials or registry data from a large, broadly representative cohort are necessary to establish the long-term safety and risk for deep fungal infections with IL-17 and especially the newer IL-23 inhibitors.
A small percentage of patients from the reviewed literature did develop superficial candidiasis. This outcome can be expected, as the central role of IL-17 and IL-23 has been recognized in immunologic protection against infections, specifically against fungi. ${ }^{11}$ Because all of the fungal infections reported for patients on IL-17 inhibitors were superficial candidiasis, guides for practical management and treatment should be implemented to standardize future research and care. A proposed screening algorithm for patients on these biologic therapies involves safety monitoring, including inspection of the oral cavity, folds, and genitals, along with inquiring about symptoms such as burning, dysgeusia, and dysuria. $^{68}$ If infection is suspected, confirmation by culture, molecular method, or optimally with esophagoscopy can be performed, and appropriate treatment may be initiated. ${ }^{68}$ Patients with candida infections of the oral cavity, folds, or genitals can be placed on topical therapy such as nystatin, amphotericin B, ciclopirox, or other azoles, while those with infections of the esophagus can be started on oral fluconazole. ${ }^{68}$

Although there were no reported cases of deep fungal infections, the theoretical risk for developing one while on IL-17 and IL-23 inhibitors may warrant further screening prior to beginning therapy. The TNF inhibitors approved for the treatment of psoriasis currently contain a black box warning for risk for disseminated and extrapulmonary histoplasmosis, coccidioidomycosis, blastomycosis, and other invasive fungal infections, which may highlight the importance of thorough evaluation and awareness of endemic areas for patients on biologics. Prior to initiating treatment with TNF inhibitors, current suggestions involve performing a thorough examination along with keeping a high index of suspicion for invasive fungal infections in patients who live in or have traveled to endemic regions. ${ }^{69}$

Screening for invasive fungal infections for patients on TNF inhibitors involves questioning about potential exposures, such as demolition of old buildings, bird roosts, or spelunking. ${ }^{70}$ Serologies or antigen testing can be used routinely, but as these tests are insensitive, empiric antifungal therapy should be initiated if there is high enough clinical suspicion. ${ }^{71}$ Currently, there are no clinical guidelines regarding fungal screening and initiation of IL-17 and IL-23 inhibitors for treatment of psoriasis and other inflammatory conditions, but careful stewardship over using these effective medications should still be practiced.

Upon review of the available safety data on the use of IL-17 and IL-23 inhibitors for the treatment of psoriasis and other inflammatory conditions, there does not appear to be an increased incidence of deep fungal infections. Physicians, however, should still be cautiously optimistic in prescribing these medications, as there is a theoretical risk for infection for all patients on biologics. A high index of suspicion for patients presenting with symptoms of fungal infections should be maintained, and 
appropriate diagnosis and management should be initiated if they do occur.

\section{REFERENCES}

1. Parisi R, Symmons DP, Griffiths CE, et al. Global epidemiology of psoriasis: a systematic review of incidence and prevalence. J Invest Dermatol. 2013;133:377-385.

2. Koo J, Marangell LB, Nakamura M, et al. Depression and suicidality in psoriasis: review of the literature including the cytokine theory of depression. J Eur Acad Dermatol Venereol. 2017;31:1999-2009.

3. Krueger JG, Bowcock A. Psoriasis pathophysiology: current concepts of pathogenesis. Ann Rheum Dis. 2005;64 (suppl 2):ii30-36.

4. Lee E, Trepicchio WL, Oestreicher JL, et al. Increased expression of interleukin 23 p19 and p40 in lesional skin of patients with psoriasis vulgaris. J Exp Med. 2004;199:125-130.

5. Lowes MA, Kikuchi T, Fuentes-Duculan J, et al. Psoriasis vulgaris lesions contain discrete populations of Th1 and Th17 T cells. J Invest Dermatol. 2008;128:1207-1211.

6. Shear NH. Fulfilling an unmet need in psoriasis: do biologicals hold the key to improved tolerability? Drug Saf. 2006;29:49-66.

7. Lee JH, Slifman NR, Gershon SK, et al. Life-threatening histoplasmosis complicating immunotherapy with tumor necrosis factor alpha antagonists infliximab and etanercept. Arthritis Rheum. 2002;46:2565-2570.

8. Leonardi C, Matheson R, Zachariae C, et al. Anti-interleukin-17 monoclonal antibody ixekizumab in chronic plaque psoriasis. $N$ Engl J Med. 2012;366:1190-1199.

9. McInnes IB, Sieper J, Braun J, et al. Efficacy and safety of secukinumab, a fully human anti-interleukin-17A monoclonal antibody, in patients with moderate-to-severe psoriatic arthritis: a 24-week, randomised, double-blind, placebo-controlled, phase II proof-of-concept trial. Ann Rheum Dis. 2014;73:349-356.

10. Papp KA, Leonardi C, Menter A, et al. Brodalumab, an anti-interleukin17-receptor antibody for psoriasis. N Engl J Med. 2012;366:1181-1189.

11. Isailovic N, Daigo K, Mantovani A, et al. Interleukin-17 and innate immunity in infections and chronic inflammation. J Autoimmun. 2015;60:1-11.

12. Bissonnette R, Luger $T$, Thaci $D$, et al. Secukinumab sustains good efficacy and favourable safety in moderate-to-severe psoriasis after up to 3 years of treatment: results from a double-blind extension study. Br J Dermatol. 2017;177:1033-1042.

13. Bissonnette R, Luger $\mathrm{T}$, Thaci $\mathrm{D}$, et al. Secukinumab demonstrates high sustained efficacy and a favourable safety profile in patients with moderate-to-severe psoriasis through 5 years of treatment (SCULPTURE Extension Study). J Eur Acad Dermatol Venereol. 2018;32:1507-1514.

14. Blauvelt A, Prinz JC, Gottlieb AB, et al. Secukinumab administration by pre-filled syringe: efficacy, safety and usability results from a randomized controlled trial in psoriasis (FEATURE). $\mathrm{Br} J$ Dermatol. 2015;172:484-493.

15. Paul C, Lacour JP, Tedremets L, et al. Efficacy, safety and usability of secukinumab administration by autoinjector/pen in psoriasis: a randomized, controlled trial (JUNCTURE). J Eur Acad Dermatol Venereol. 2015;29:1082-1090

16. Bagel J, Duffin KC, Moore A, et al. The effect of secukinumab on moderate-to-severe scalp psoriasis: Results of a 24 -week, randomized, double-blind, placebo-controlled phase 3b study. J Am Acad Dermatol. 2017;77:667-674.

17. Blauvelt A, Reich K, Tsai TF, et al. Secukinumab is superior to ustekinumab in clearing skin of subjects with moderate-to-severe plaque psoriasis up to 1 year: results from the CLEAR study. J Am Acad Dermatol. 2017;76:60.e9-69.e9.

18. Thaci D, Blauvelt A, Reich $\mathrm{K}$, et al. Secukinumab is superior to ustekinumab in clearing skin of subjects with moderate to severe plaque psoriasis: CLEAR, a randomized controlled trial. J Am Acad Dermatol. 2015;73:400-409.

19. Gottlieb A, Sullivan J, van Doorn M, et al. Secukinumab shows significant efficacy in palmoplantar psoriasis: results from GESTURE, a randomized controlled trial. J Am Acad Dermatol. 2017;76:70-80
20. Ohtsuki M, Morita A, Abe M, et al. Secukinumab efficacy and safety in Japanese patients with moderate-to-severe plaque psoriasis: subanalysis from ERASURE, a randomized, placebo-controlled, phase 3 study. J Dermatol. 2014;41:1039-1046.

21. Wu NL, Hsu CJ, Sun FJ, et al. Efficacy and safety of secukinumab in Taiwanese patients with moderate to severe plaque psoriasis: subanalysis from ERASURE phase III study. J Dermatol. 2017;44:1129-1137.

22. Papp KA, Langley RG, Sigurgeirsson B, et al. Efficacy and safety of secukinumab in the treatment of moderate-to-severe plaque psoriasis: a randomized, double-blind, placebo-controlled phase II dose-ranging study. Br J Dermatol. 2013;168:412-421.

23. Rich P, Sigurgeirsson B, Thaci D, et al. Secukinumab induction and maintenance therapy in moderate-to-severe plaque psoriasis: a randomized, double-blind, placebo-controlled, phase II regimen-finding study. Br J Dermatol. 2013;168:402-411.

24. Kavanaugh A, Mease PJ, Reimold AM, et al. Secukinumab for long-term treatment of psoriatic arthritis: a two-year followup from a phase III, randomized, double-blind placebo-controlled study. Arthritis Care Res (Hoboken). 2017;69:347-355

25. McInnes IB, Mease PJ, Kirkham B, et al. Secukinumab, a human antiinterleukin-17A monoclonal antibody, in patients with psoriatic arthritis (FUTURE 2): a randomised, double-blind, placebo-controlled, phase 3 trial. Lancet. 2015;386:1137-1146.

26. Nash P, Mease PJ, McInnes IB, et al. Efficacy and safety of secukinumab administration by autoinjector in patients with psoriatic arthritis: results from a randomized, placebo-controlled trial (FUTURE 3). Arthritis Res Ther. 2018;20:47.

27. Sticherling M, Mrowietz $U$, Augustin M, et al. Secukinumab is superior to fumaric acid esters in treating patients with moderate-to-severe plaque psoriasis who are naive to systemic treatments: results from the randomized controlled PRIME trial. Br J Dermatol. 2017;177:1024-1032.

8. Braun J, Baraliakos X, Deodhar A, et al. Effect of secukinumab on clinical and radiographic outcomes in ankylosing spondylitis: 2-year results from the randomised phase III MEASURE 1 study. Ann Rheum Dis. 2017:76:1070-1077.

29. Marzo-Ortega H, Sieper J, Kivitz A, et al. Secukinumab provides sustained improvements in the signs and symptoms of active ankylosing spondylitis with high retention rate: 3-year results from the phase III trial, MEASURE 2. RMD Open. 2017;3:e000592.

30. Pavelka K, Kivitz A, Dokoupilova E, et al. Efficacy, safety, and tolerability of secukinumab in patients with active ankylosing spondylitis: a randomized, double-blind phase 3 study, MEASURE 3. Arthritis Res Ther. 2017;19:285

31. Callis Duffin K, Bagel J, Bukhalo M, et al. Phase 3, open-label, randomized study of the pharmacokinetics, efficacy and safety of ixekizumab following subcutaneous administration using a prefilled syringe or an autoinjector in patients with moderate-to-severe plaque psoriasis (UNCOVER-A). J Eur Acad Dermatol Venereol. 2017;31:107-113.

32. Gordon KB, Colombel JF, Hardin DS. Phase 3 trials of ixekizumab in moderate-to-severe plaque psoriasis. N Engl J Med. 2016;375:2102.

33. Saeki $\mathrm{H}$, Nakagawa $\mathrm{H}$, Nakajo K, et al. Efficacy and safety of ixekizumab treatment for Japanese patients with moderate to severe plaque psoriasis, erythrodermic psoriasis and generalized pustular psoriasis: results from a 52-week, open-label, phase 3 study (UNCOVER-J). J Dermatol. 2017:44:355-362.

34. Reich K, Pinter A, Lacour JP, et al. Comparison of ixekizumab with ustekinumab in moderate-to-severe psoriasis: 24-week results from IXORA-S, a phase III study. Br J Dermatol. 2017;177:1014-1023.

35. Zachariae C, Gordon K, Kimball AB, et al. Efficacy and safety of ixekizumab over 4 years of open-label treatment in a phase 2 study in chronic plaque psoriasis. J Am Acad Dermatol. 2018;79:294.e6-301.e6.

36. van der Heijde D, Gladman DD, Kishimoto M, et al. Efficacy and safety of ixekizumab in patients with active psoriatic arthritis: 52-week results from a phase III study (SPIRIT-P1). J Rheumatol. 2018;45:367-377.

37. van der Heijde D, Cheng-Chung Wei J, Dougados M, et al. Ixekizumab, an interleukin-17A antagonist in the treatment of ankylosing spondylitis or radiographic axial spondyloarthritis in patients previously untreated with biological disease-modifying antirheumatic drugs (COAST-V): 16 week results of a phase 3 randomised, 
double-blind, active-controlled and placebo-controlled trial. Lancet. 2018;392:2441-2451.

38. Nakagawa $\mathrm{H}$, Niiro $\mathrm{H}$, Ootaki $\mathrm{K}$, et al. Brodalumab, a human antiinterleukin-17-receptor antibody in the treatment of Japanese patients with moderate-to-severe plaque psoriasis: efficacy and safety results from a phase II randomized controlled study. J Dermatol Sci. 2016;81:44-52

39. Umezawa $Y$, Nakagawa $H$, Niiro $H$, et al. Long-term clinical safety and efficacy of brodalumab in the treatment of Japanese patients with moderate-to-severe plaque psoriasis. J Eur Acad Dermatol Venereol. 2016;30:1957-1960.

40. Papp KA, Reich K, Paul C, et al. A prospective phase III, randomized, double-blind, placebo-controlled study of brodalumab in patients with moderate-to-severe plaque psoriasis. $\mathrm{Br} J$ Dermatol. 2016;175:273-286.

41. Papp K, Leonardi C, Menter A, et al. Safety and efficacy of brodalumab for psoriasis after 120 weeks of treatment. J Am Acad Dermatol. 2014;71:1183.e3-1190.e3

42. Yamasaki K, Nakagawa H, Kubo Y, et al. Efficacy and safety of brodalumab in patients with generalized pustular psoriasis and psoriatic erythroderma: results from a 52-week, open-label study. Br J Dermatol. 2017;176:741-751.

43. Mease PJ, Genovese MC, Greenwald MW, et al. Brodalumab, an anti-IL17RA monoclonal antibody, in psoriatic arthritis. N Engl J Med. 2014;370:2295-2306

44. Martin DA, Churchill M, Flores-Suarez L, et al. A phase Ib multiple ascending dose study evaluating safety, pharmacokinetics, and early clinical response of brodalumab, a human anti-IL-17R antibody, in methotrexate-resistant rheumatoid arthritis. Arthritis Res Ther. 2013;15:R164.

45. Busse WW, Holgate S, Kerwin E, et al. Randomized, double-blind, placebo-controlled study of brodalumab, a human anti-IL-17 receptor monoclonal antibody, in moderate to severe asthma. Am J Respir Crit Care Med. 2013;188:1294-1302.

46. Igarashi A, Kato T, Kato M, et al. Efficacy and safety of ustekinumab in Japanese patients with moderate-to-severe plaque-type psoriasis: longterm results from a phase 2/3 clinical trial. J Dermatol. 2012;39:242-252.

47. Krueger GG, Langley RG, Leonardi C, et al. A human interleukin-12/23 monoclonal antibody for the treatment of psoriasis. $N$ Engl J Med. 2007;356:580-592.

48. Leonardi CL, Kimball AB, Papp KA, et al. Efficacy and safety of ustekinumab, a human interleukin-12/23 monoclonal antibody, in patients with psoriasis: 76-week results from a randomised, double-blind, placebo-controlled trial (PHOENIX 1). Lancet. 2008;371:1665-1674.

49. Tsai TF, Ho JC, Song M, et al. Efficacy and safety of ustekinumab for the treatment of moderate-to-severe psoriasis: a phase III, randomized, placebo-controlled trial in Taiwanese and Korean patients (PEARL). J Dermatol Sci. 2011;63:154-163.

50. Gordon KB, Strober B, Lebwohl M, et al. Efficacy and safety of risankizumab in moderate-to-severe plaque psoriasis (UltIMMa-1 and UltIMMa-2): results from two double-blind, randomised, placebocontrolled and ustekinumab-controlled phase 3 trials. Lancet. 2018;392:650-661.

51. Krueger JG, Ferris LK, Menter A, et al. Anti-IL-23A mAb BI 655066 for treatment of moderate-to-severe psoriasis: safety, efficacy, pharmacokinetics, and biomarker results of a single-rising-dose, randomized, double-blind, placebo-controlled trial. J Allergy Clin Immunol. 2015;136:116.e7-124.e7.

52. Ohtsuki M, Fujita H, Watanabe M, et al. Efficacy and safety of risankizumab in Japanese patients with moderate to severe plaque psoriasis: results from the SustaIMM phase 2/3 trial. J Dermatol. 2019;46:686-694.

53. Papp KA, Blauvelt A, Bukhalo $\mathrm{M}$, et al. Risankizumab versus ustekinumab for moderate-to-severe plaque psoriasis. N Engl J Med. 2017;376:1551-1560.

54. Reich K, Gooderham M, Thaci D, et al. Risankizumab compared with adalimumab in patients with moderate-to-severe plaque psoriasis
(IMMvent): a randomised, double-blind, active-comparator-controlled phase 3 trial. Lancet. 2019;394:576-586.

55. Blauvelt A, Papp KA, Griffiths CE, et al. Efficacy and safety of guselkumab, an anti-interleukin-23 monoclonal antibody, compared with adalimumab for the continuous treatment of patients with moderate to severe psoriasis: results from the phase III, double-blinded, placeboand active comparator-controlled VOYAGE 1 trial. J Am Acad Dermatol. 2017;76:405-417.

56. Deodhar $\mathrm{A}$, Gottlieb $\mathrm{AB}$, Boehncke $\mathrm{WH}$, et al. Efficacy and safety of guselkumab in patients with active psoriatic arthritis: a randomised, double-blind, placebo-controlled, phase 2 study. Lancet. 2018;391:2213-2224.

57. Gordon KB, Duffin KC, Bissonnette R, et al. A phase 2 trial of guselkumab versus adalimumab for plaque psoriasis. $N$ Engl J Med. 2015;373:136-144.

58. Langley RG, Tsai TF, Flavin S, et al. Efficacy and safety of guselkumab in patients with psoriasis who have an inadequate response to ustekinumab: results of the randomized, double-blind, phase III NAVIGATE trial. Br J Dermatol. 2018;178:114-123.

59. Nemoto O, Hirose K, Shibata S, et al. Safety and efficacy of guselkumab in Japanese patients with moderate-to-severe plaque psoriasis: a randomized, placebo-controlled, ascending-dose study. Br J Dermatol. 2018;178:689-696.

60. Ohtsuki M, Kubo H, Morishima H, et al. Guselkumab, an antiinterleukin-23 monoclonal antibody, for the treatment of moderate to severe plaque-type psoriasis in Japanese patients: Efficacy and safety results from a phase 3, randomized, double-blind, placebo-controlled study. J Dermatol. 2018;45:1053-1062.

61. Reich K, Armstrong AW, Foley P, et al. Efficacy and safety of guselkumab, an anti-interleukin-23 monoclonal antibody, compared with adalimumab for the treatment of patients with moderate to severe psoriasis with randomized withdrawal and retreatment: results from the phase III, double-blind, placebo- and active comparator-controlled VOYAGE 2 trial. J Am Acad Dermatol. 2017;76:418-431.

62. Reich K, Armstrong AW, Langley RG, et al. Guselkumab versus secukinumab for the treatment of moderate-to-severe psoriasis (ECLIPSE): results from a phase 3, randomised controlled trial. Lancet. 2019;394:831-839.

63. Terui T, Kobayashi S, Okubo $Y$, et al. Efficacy and safety of guselkumab, an anti-interleukin 23 monoclonal antibody, for palmoplantar pustulosis: a randomized clinical trial. JAMA Dermatol. 2018;154:309-316.

64. Papp K, Thaci D, Reich K, et al. Tildrakizumab (MK-3222), an anti-interleukin-23p19 monoclonal antibody, improves psoriasis in a phase Ilb randomized placebo-controlled trial. $\mathrm{Br} J$ Dermatol. 2015;173:930-939.

65. Reich K, Papp KA, Blauvelt A, et al. Tildrakizumab versus placebo or etanercept for chronic plaque psoriasis (reSURFACE 1 and reSURFACE 2): results from two randomised controlled, phase 3 trials. Lancet. 2017;390:276-288.

66. Gordon KB, Papp KA, Langley RG, et al. Long-term safety experience of ustekinumab in patients with moderate to severe psoriasis (part II of II): results from analyses of infections and malignancy from pooled phase II and III clinical trials. J Am Acad Dermatol. 2012;66:742-751.

67. Papp KA, Griffiths CE, Gordon K, et al. Long-term safety of ustekinumab in patients with moderate-to-severe psoriasis: final results from 5 years of follow-up. Br J Dermatol. 2013;168:844-854.

68. Saunte DM, Mrowietz U, Puig L, et al. Candida infections in patients with psoriasis and psoriatic arthritis treated with interleukin-17 inhibitors and their practical management. Br J Dermatol. 2017;177:47-62.

69. Lis K, Kuzawinska O, Balkowiec-Iskra E. Tumor necrosis factor inhibitors—state of knowledge. Arch Med Sci. 2014;10:1175-1185.

70. Hage CA, Bowyer S, Tarvin SE, et al. Recognition, diagnosis, and treatment of histoplasmosis complicating tumor necrosis factor blocker therapy. Clin Infect Dis. 2010;50:85-92

71. Hage CA, Ribes JA, Wengenack NL, et al. A multicenter evaluation of tests for diagnosis of histoplasmosis. Clin Infect Dis. 2011;53:448-454. 


\section{APPENDIX}

eTABLE. IL-12/IL-23 and IL-23 Inhibitors Exposure Data Summary

\begin{tabular}{|c|c|c|c|c|c|c|}
\hline Reference & Year & Country & Biologic & $\begin{array}{l}\text { Patients Exposed to } \\
\text { Biologic, } n\end{array}$ & Data Source & $\begin{array}{l}\text { Reported Deep Fungal } \\
\text { Infections, } \mathrm{n}\end{array}$ \\
\hline Igarashi et al ${ }^{46}$ & 2012 & Japan & Ustekinumab & 126 & RCT & 0 \\
\hline Krueger et al| ${ }^{47}$ & 2007 & International & Ustekinumab & 256 & RCT & 0 \\
\hline Leonardi et al ${ }^{48}$ & 2008 & International & Ustekinumab & 511 & RCT & 0 \\
\hline Tsai et al ${ }^{49}$ & 2011 & International & Ustekinumab & 61 & RCT & 0 \\
\hline Gordon et $a^{50}$ & 2018 & International & Risankizumab & 598 & RCT & 0 \\
\hline Krueger et $\mathrm{a}^{51}$ & 2015 & International & Risankizumab & 31 & RCT & 0 \\
\hline Ohtsuki et al $\left.\right|^{52}$ & 2019 & Japan & Risankizumab & 113 & RCT & 0 \\
\hline Papp et $a^{53}$ & 2017 & International & Risankizumab & 126 & Clinical trial & 0 \\
\hline Reich et $\mathrm{a}^{54}$ & 2019 & International & Risankizumab & 301 & Clinical trial & 0 \\
\hline Blauvelt et a ${ }^{55}$ & 2017 & International & Guselkumab & 329 & RCT & 0 \\
\hline Deodhar et al ${ }^{56}$ & 2018 & International & Guselkumab & 100 & RCT & 0 \\
\hline Gordon et $\mathrm{a}^{57}$ & 2015 & International & Guselkumab & 825 & $\mathrm{RCT}$ & 0 \\
\hline Langley et al ${ }^{58}$ & 2018 & International & Guselkumab & 135 & Clinical trial & 0 \\
\hline Nemoto et $a^{59}$ & 2018 & Japan & Guselkumab & 20 & RCT & 0 \\
\hline Ohtsuki et $a^{60}$ & 2018 & Japan & Guselkumab & 128 & RCT & 0 \\
\hline Reich et al ${ }^{61}$ & 2017 & International & Guselkumab & 496 & RCT & 0 \\
\hline Reich et a ${ }^{62}$ & 2019 & International & Guselkumab & 534 & Clinical trial & 0 \\
\hline Terui et al ${ }^{63}$ & 2018 & Japan & Guselkumab & 25 & $\mathrm{RCT}$ & 0 \\
\hline Papp et al ${ }^{64}$ & 2015 & International & Tildrakizumab & 309 & $\mathrm{RCT}$ & 0 \\
\hline Reich et al ${ }^{65}$ & 2017 & International & Tildrakizumab & 617 & $\mathrm{RCT}$ & 0 \\
\hline
\end{tabular}

Abbreviation: $\mathrm{RCT}$, randomized controlled trial. 